Ann. Zootech., r979, 28 (4), 443-452.

\title{
Influence de l'âge au premier accouplement sur la fertilité de jeunes lapines et leurs performances en première portée
}

\author{
Lea VAN DEN BROECK et PH. LAMPO \\ avec la collaboration technique de H. DENoO
}

Leerstoel dierlijke genetica en Veeteelt

Faculteit diergeneeshunde, Rijksuniversiteit-Gent Heidestraat 1.9,922o Merelbeke (Belgique)

\begin{abstract}
Résumé
Cet essai étudie l'influence de l'âge à la première saillie sur la réceptivité et la fertilité des lapines ainsi que sur les performances de la première portée. 2ro lapines de la race Blanc de Termonde ont été utilisées, dont 70 sont saillies pour la première fois à 4 mois, autant à 4 mois I/2 et autant à 5 mois, après un flushing de 4 jours. Deux niveaux alimentaires (I 50 ou $200 \mathrm{~g}$ d'aliment/jour) ont également été étudiés.

L'essai montre que les résultats obtenus pour les paratnètres mesurés sont généralement acceptables lorsque le niveau alimentaire est correct; seule la mortalité des lapereaux entre la naissance et le sevrage est dans tous les cas très élevée.
\end{abstract}

\section{Introduction}

L'âge d'apparition de la puberté et les résultats du premier accouplement des jeunes lapines varient en fonction de nombreux facteurs externes. Le climat, la saison, la race, l'alimentation, l'éclairement ainsi que des différences individuelles ont été indiqués (Critchlov et BAR SELA, I967, MCDONAL,D, I975).

Le facteur déterminant semble être le métabolisme basal qui diminue dès la naissance et tend vers une valeur critique, moment où la puberté commence (LEVASSEUR, I977).

Selon Martinet, (I973) et Prud'hon (I975) l'apparition des premiers follicules à antrum se situerait à 9 -Io semaines et la puberté entre 5 et 8 mois.

Toutefois les travaux de LEBAS (I974) et TORRES (I977) montrent que des 
taux d'ovulation et de fertilité très satisfaisants sont obtenus dès 1'âge de quatre mois.

Les travaux de LEFEvre, MarTINeT et MoreT (I976) ainsi que ceux de LEFEVRE et MORET (I978) mettent en évidence l'influence de changements des conditions de milieu sur l'ovulation tandis que nous avons obtenu des résultats avec un flushing de 4 jours précédant la présentation aut mâle (VAN DEN BROECK, LAMPO, I977).

En ce qui concerne les performances des lapines primipares les résultats sont rares et contradictoires.

Selon Rouvier, Poujardieu et Vrillon (I973) les effectifs des premières portées seraient de Io $\mathrm{p}$. Ioo inférieurs et les taux de mortalité de I3 à 2 I p. IOO supérieurs aux données correspondantes des portées ultérieures. KALINowsKi et RUDOLF (I977), sur Néozélandaises blanches comptent 4,2 lapereaux sevrés à 35 jours en premières portée contre 5,0 pour la moyenne des portées 2 à 6 . LEBAs travaillant sur des lapines saillies à 4 mois, 4 mois $I / 2$ ou 5 mois obtient des effectifs au sevrage ( 28 jours) allant de 7 à 8 .

Dans le présent travail, nous nous sommes proposés d'étudier l'influence de 1'âge au premier accouplement et du niveau d'alimentation sur la fertilité des lapines et leurs performances en première portée.

\section{Matériel et méthodes}

\section{A. - Bâtiment}

Une description de nos installations a été donnée antérieurement (VAN DEN BROECK, LAMPO I977).

\section{B. - Animanx}

Les 2 Io lapines retenues sont de la race Blanc de Termonde d'un poids moyen en production de $4940 \mathrm{~g} \pm 479,0 \mathrm{~g}$. Elles doivent satisfaire aux exigences suivantes : avoir un poids minimum de $2500 \mathrm{~g}$ à 1 'âge maximum de 77 jours, et ne pas dépasser l'indice de consommation de 4 pendant l'engraissement. Le sevrage se fait à 3239 jours. Les lapines sont mises en cages individuelles, et réparties au hasard en trois groupes. Les femelles du premier groupe sont accouplées à 1'âge de 4 mois. Celles du deuxième groupe à 4 mois et demi, les autres à 1'âge de 5 mois. La première présentation, au jour $o$, se fait après un flushing de 4 jours. S'il n'y a pas de saillie, le flushing continue jusqu'à la saillie au jour 3,7 , Io ou $\mathrm{x} 4$. Dix ou onze jours après la mise-bas, les lapines sont réaccouplées. Si les petits meurent plus tôt, la lapine est réaccouplée à la première occasion. Le contrôle de gestation est fait par palpation, $x_{4}$ ou $I_{7}$ jours après la saillie. Les données ont été recueillies de novembre I975 jusqu'à octobre I978. Ia répartition des lapines au cours des saisons figure au tableau $\mathrm{I}$. 
TABLEAU I

Répartition des lapines selon le jour de la sélection

Distribution of the does by order of selection day

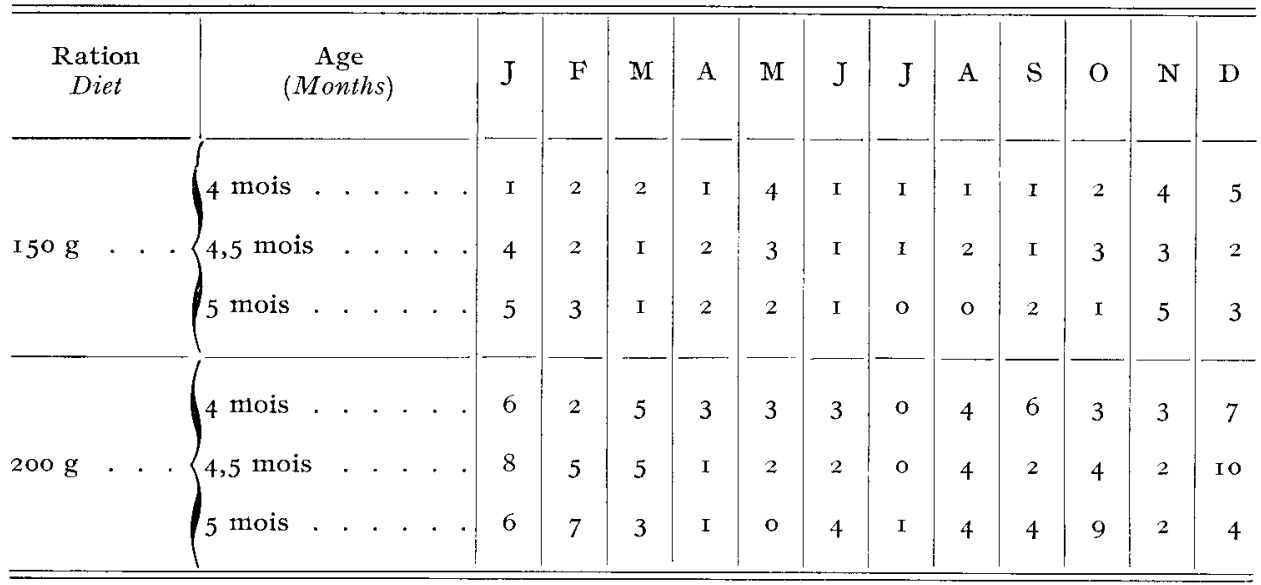

\section{C. - Alimentation}

L'aliment distribué est un granulé commercial dosant $18,5 \mathrm{p}$. Ioo de matières azotées, 4,2 p. Ioo de matières grasses, $47 \mathrm{p}$. Ioo de sucres et amidon et II,5 p. Ioo de cellulose brute; sa valeur énergétique est d'environ $2700 \mathrm{Kcal}$ métabolisables.

Jusqu'en décembre I 976 les lapines recevaient I50 g de granulé par jour. Compte tenu de nos travaux précédents (VAN DEN BROECK, LAMPO, I977) cette quantité a été portée à $200 \mathrm{~g}$ à partir de janvier 1977 afin d'assurer aux lapines une croissance suffisante.

La ration est distribuée quotidiennement à l'exception du vendredi où l'on donne trois rations en prévision du week-end. Il est tenu compte des quantités restant dans les trémies lors de la distribution d'aliment. Le rationnement est abandonné deux jours avant la mise bas, et remplacé par une alimentation libre.

\section{D. - Méthodes statistiques}

Les résultats ont été soumis au test Chi carré et à l'analyse de la Variance, sans aucune transformation préalable des données.

\section{Résultats}

\section{A. - Poids à la $I^{\text {re }}$ saillie}

Des résultats, reproduits dans le tableau 2, il ressort qu'il y a eu des différences significatives entre les poids des lapines nourries à différents niveaux, et cela à chaque âge considéré. Il existe aussi des différences entre âges dans chaque régime, 
TABLEAU 2

Poids à la ${ }_{I}{ }^{\mathrm{re}}$ resaillie

Weight at first mating

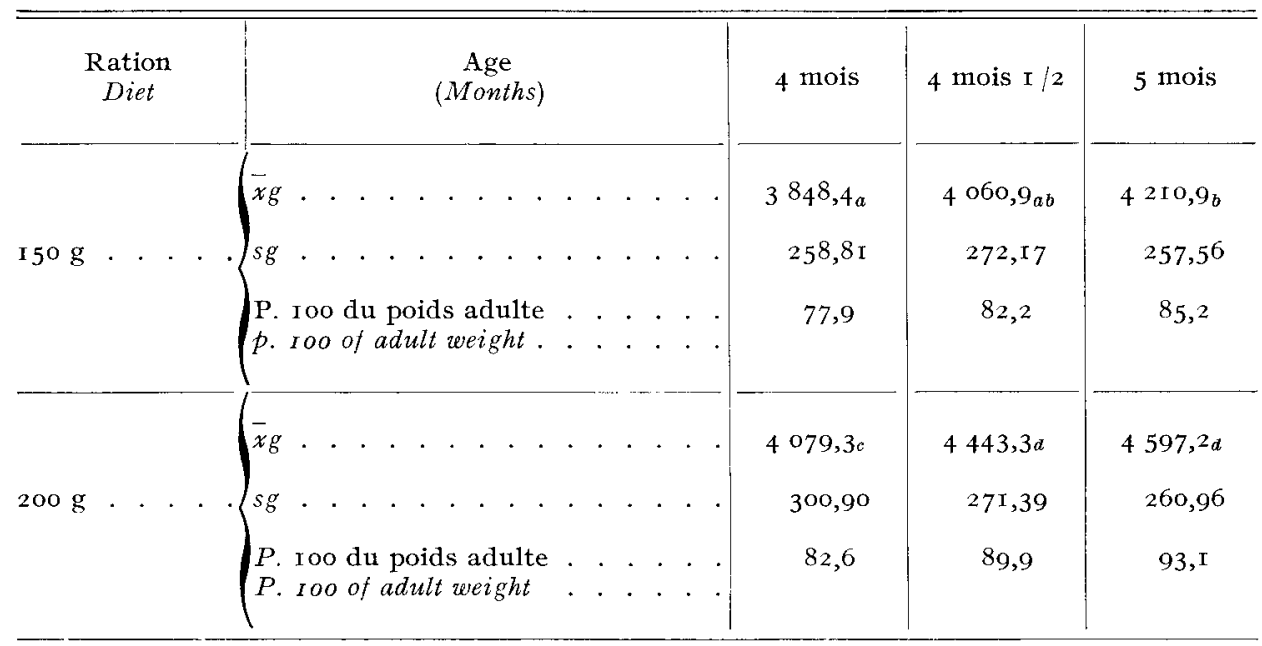

Les chiffres affectés de la même lettre ne diffèrent pas significativement (There is no significant difference between figures followed by the same letter).

mais elles sont moins prononcées. La différence de poids entre deux groupes d'âge diminue avec l'âge, d'une façon plus nette pour le régime de $200 \mathrm{~g}$ que pour celui de $5^{\circ} \mathrm{g}$. Notons qu'il s'agit des poids notés après au moins 4 jours de consommation libre.

\section{B. - Consommation d'aliment}

Les jeunes lapines mangent à peu près toute la ration distribuée. Environ $3 \mathrm{p}$. roo des rations de $150 \mathrm{~g}$ et $5 \mathrm{p}$. Ioo seulement des rations de $200 \mathrm{~g}$ étaient refusés. Il n'y a pas de différences entre âges, la différence entre les rations par contre, est évidente (tabl. 3).

Considérant la prise d'aliment pendant le flushing, nous remarquons que les lapines du régime de $5^{\circ} \mathrm{g}$ tendent à rattraper leur retard, en mangeant plus du double de leur ration initiale.

Les lapines qui avaient reçu $200 \mathrm{~g}$ par jour, mangent à peu près I40 p. Ioo de leur ration normale, et significativement moins que celles du régime $150 \mathrm{~g}$ (tabl. 3). 
TABLEAU 3

Consommation d'aliment pendant la période d'élevage et le flushing Feed intake during rearing and fushing

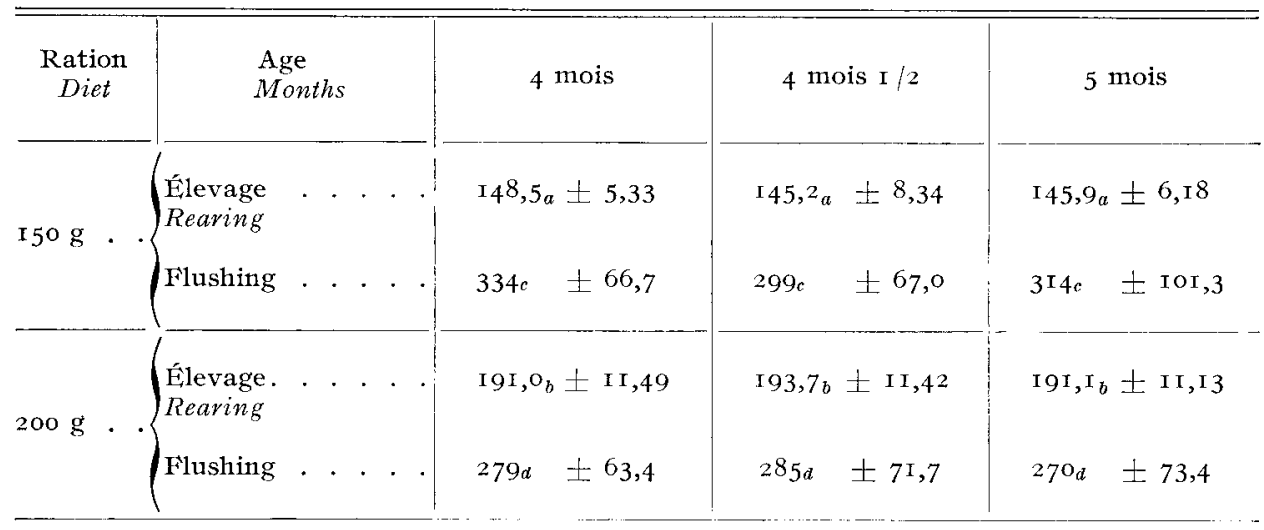

Les chiffres affectés de la même lettre ne diffèrent pas significativement. (There is no significant difference between figures followed by the same letter).

\section{C. - Efficacité de l'accouplement}

Les résultats concernant l'efficacité de l'accouplement sont donnés dans le tableau 4 .

Dans tous les groupes, exception faite du groupe I50 g à 4 mois et demi, plus de trois quarts des femelles acceptent le mâle dès la première présentation. Si 1'on attend encore quelques jours, (tenant compte du cycle ovarien peut-être en pro- ou " met " oestrus) plus de 95 p. roo des femelles acceptent l'accouplement. Dans le groupe r5o g 4 mois et demi, il n'y a que 84 p. Ioo de réponse. Les différences entre âges, intra ration, ne sont pas statistiquement significatives. Par contre, la différence entre rations est significative, sauf pour les lapines de 4 mois. Après quelques jours, ces différences disparaissent.

\section{D. - Taux de gestation et qualité de la mise-bas}

Les résultats obtenus pour ces critères sont repris dans le tableau 4. La comparaison des taux de gestation indique une différence significative entre les rationnements, en faveur de la ration $200 \mathrm{~g}$.

D'autre part, les lapines de 4 mois sont significativement moins réceptives que les lapines plus âgées. La qualité de la mise-bas est exprimée par deux critères. Le soin du nid est qualifié de bon, lorsque les petits sont posés dans un nid de paille avec au moins un peu de poils; mauvais lorsque les lapereaux sont mal soignés, dans un nid rudimentaire ou sans nid du tout. L'allaitement au moment de la mise bas est jugé à l'aspect de repletion des ventres des lapereaux. On peut conclure que la qualité des nids et de l'allaitement aut moment de la mise-bas est signi- 


\begin{tabular}{|c|c|c|c|c|c|c|c|c|c|}
\hline \multirow{13}{*}{ 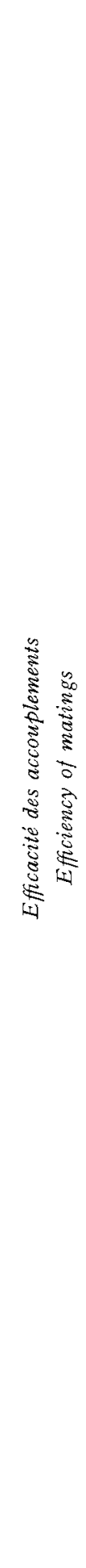 } & \multirow{4}{*}{ 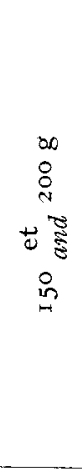 } & $\begin{array}{l}\bar{\pi} \\
\stackrel{\overrightarrow{0}}{0} \\
\dot{0}\end{array}$ & 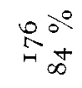 & $\begin{array}{ll}0^{2} \\
8 \\
i\end{array}$ & $\begin{array}{l}80 \\
80 \\
0\end{array}$ & $\underset{\sim}{\mathrm{m}_{\infty}^{\circ}}$ & $\begin{array}{l}^{\circ} \\
\Rightarrow 0\end{array}$ & $\frac{1}{2}$ & \multirow{13}{*}{ 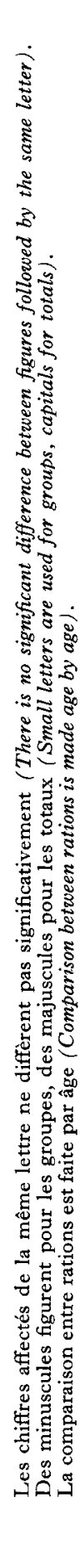 } \\
\hline & & $\begin{array}{l}\frac{n a}{3} \\
\text { in }\end{array}$ & $\begin{array}{c}-20 \\
68\end{array}$ & $\begin{array}{l}\text { i } 20 \\
8 \%\end{array}$ & $\begin{array}{c}i_{\infty} 20 \\
i_{\infty}^{\infty} \\
i_{\infty}\end{array}$ & 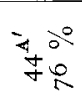 & 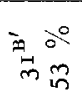 & $\begin{array}{l}\dot{m}_{10} \\
\text { m }\end{array}$ & \\
\hline & & 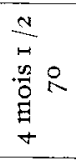 & $\begin{array}{l}2^{\circ} \\
\text { in } \\
\text { in }\end{array}$ & उ० & 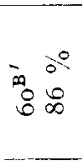 & $\begin{array}{l}\alpha^{2} \\
i n \\
\ln \infty\end{array}$ & $\begin{array}{l}x^{2} \\
\frac{m}{7} i^{2}\end{array}$ & $\begin{array}{l}00 \\
78 \\
7\end{array}$ & \\
\hline & & $\begin{array}{l}\stackrel{n}{0} \stackrel{0}{g} \\
+ \\
+\end{array}$ & $\begin{array}{l}i^{\circ} \\
80\end{array}$ & 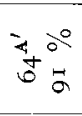 & $\begin{array}{l}400 \\
\dot{4} 0 \\
+\infty \\
+\infty\end{array}$ & 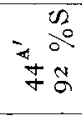 & $\begin{array}{l}\dot{y} \alpha^{\circ} \\
\hat{m}_{\infty}^{\circ}\end{array}$ & $\begin{array}{l}40^{\circ} \\
\dot{m}_{\infty}\end{array}$ & \\
\hline & & $\begin{array}{l}\bar{\pi} n \\
\stackrel{0}{0} m \\
F\end{array}$ & $\begin{array}{l}\infty_{\infty} 0^{\circ} \\
=0 \\
=0\end{array}$ & $\begin{array}{l}400 \\
m a \\
\cdots\end{array}$ & $\begin{array}{l}m_{\infty}^{\infty} \infty^{0} \\
=\infty^{+}\end{array}$ & $\begin{array}{l}40 \\
0 \\
0\end{array}$ & 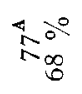 & $\stackrel{4}{\infty} 2^{\infty}$ & \\
\hline & 20 & $\begin{array}{l}\frac{\infty}{0} \\
\text { 年 } \\
\text { in }\end{array}$ & $\stackrel{\circ}{+}$ & $\begin{array}{l}20 \\
\text { : } \\
\text { 욤 }\end{array}$ & $\begin{array}{l}\stackrel{2}{\circ} \\
\square \infty\end{array}$ & 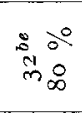 & 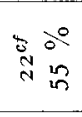 & $\begin{array}{l}\stackrel{0}{\circ} 00 \\
\stackrel{N}{N} \text { in }\end{array}$ & \\
\hline & 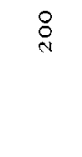 & 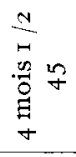 & 总 & $\begin{array}{l}\text { : } 20 \\
m 8\end{array}$ & $\begin{array}{l}: 00 \\
\stackrel{0}{0} \\
+\infty\end{array}$ & $\begin{array}{l}\text { : } 00 \\
0 \\
\end{array}$ & 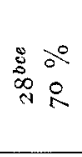 & 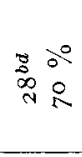 & \\
\hline & & 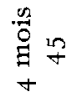 & $\begin{array}{l}: 00 \\
: 00 \\
+\infty\end{array}$ & $\begin{array}{l}: 00 \\
\text { is }\end{array}$ & $\begin{array}{l}: \partial^{\circ} \\
m n\end{array}$ & 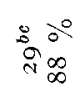 & 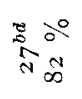 & 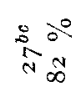 & \\
\hline & & $\stackrel{\pi}{\stackrel{\pi}{0} \ltimes ~}$ & $\begin{array}{l}4^{2} \\
m^{2}\end{array}$ & $\begin{array}{l}400 \\
8 \sigma a \\
\sigma\end{array}$ & $\ln ^{4} \partial^{\circ}$ & $\begin{array}{l}40^{\circ} \\
48\end{array}$ & $\frac{a^{\circ}}{\mathrm{m}^{\circ}}$ & $\begin{array}{l}400 \\
+\infty \\
+\infty\end{array}$ & \\
\hline & $\infty$ & $\begin{array}{l}\text { 象 } \\
\text { 吾 } \\
\text { in }\end{array}$ & \begin{tabular}{l}
00 \\
\hdashline \\
$=$
\end{tabular} & 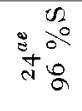 & 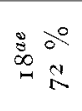 & \begin{tabular}{l}
$: 00$ \\
\multirow{4}{*}{} \\
\multirow{6}{*}{}
\end{tabular} & $\begin{array}{l}\text { o } \\
\text { o }\end{array}$ & 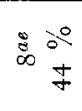 & \\
\hline & in & 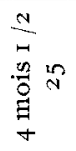 & $\begin{array}{l}20 \\
\text { to } \\
\forall \text { in }\end{array}$ & $\begin{array}{l}\mathbb{a} \\
\vec{*}\end{array} \frac{2}{\infty}$ & 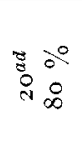 & $\begin{array}{l}\text { 형ㅇ } \\
\text { in }\end{array}$ & $\begin{array}{l}\underbrace{}_{1} \\
i n \\
n\end{array}$ & 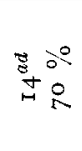 & \\
\hline & & 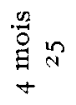 & $\begin{array}{l}: 00 \\
: 0 \\
0\end{array}$ & \begin{tabular}{l}
$: 20$ \\
\multirow{5}{*}{8}
\end{tabular} & $\begin{array}{l}\mathrm{s}_{0}^{\circ} \\
\mathrm{n}\end{array}$ & $\begin{array}{l}\text { is } \\
\text { in } 8 \\
\text { i }\end{array}$ & $\begin{array}{l}\mathbb{Z} \\
\mathbb{N}:\end{array}$ & 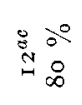 & \\
\hline & & 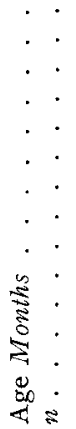 & 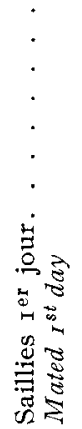 & 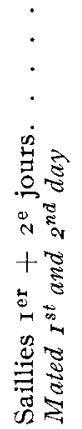 & 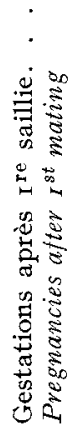 & 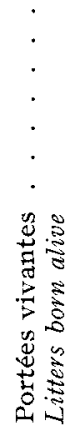 & 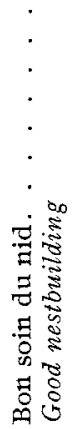 & 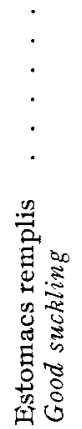 & \\
\hline
\end{tabular}


ficativement moins bonne pour les lapines de 5 mois. Pour ces deux critères, il n'y a pas de différence significative ni entre les rations, ni intra ration, mais il semble y avoir une tendance à la diminution de 4 mois à 5 mois, pour chaque critère et dans chaque ration.

\title{
E. - Performances des premières portées
}

Les performances des premières portées figurent au tableau 5. Si les effectifs nés totaux sont élevés, les effectifs nés vivants sont plus réduits, notamment dans le lot rationné à $\mathrm{I} 50 \mathrm{~g}$. La mortalité entre la naissance et le sevrage est très élevée dans tous les lots, le pourcentage de lapereaux sevrés étant toujours inférieur à 50 p. roo des nés totaux.

S'il 1'on ne tient compte que des portées vivantes (tabl. 6) les effectifs sont un peu plus élevés et ne varient pas avec l'âge ni avec le niveau d'alimentation. Les caractéristiques de croissance sont comparables.

\section{Conclusion}

La présente étude montre essentiellement que les femelles ont une ardeur sexuelle satisfaisante quel que soit l'âge considéré à la première saillie, à condition que le niveau d'alimentation soit correct. De même le taux de gestation est très acceptable. Les qualités maternelles des mères ne semblent pas affectées par une saillie précoce et paraissent même meilleures pour les lapines saillies à 4 mois que pour celles saillies à 5 mois. Cependant, dans tous les cas, la mortalité entre la naissance et le sevrage est très élevée. Ces résultats sont dans 1'ensemble en accord avec ceux obtenus par LEBAS (I974) avec des lapines croisées Néozélandais Californien. Il semble donc, que dans l'intervalle étudié, 1'âge à la première saillie n'a qu'une incidence secondaire sur la productivité des lapines à cette première saillie.

Toutefois, LEBAS (I974) a montré que sur un an de production, les performances des lapines saillies à 4 mois $\mathrm{I} / 2$ sont meilleures que celles des lapines saillies plus précocement. Les causes de la mortalité élevée observée chez les lapereaux restent à étudier.

Accepté pour publication en juin 1979.

\section{Summary}

\author{
Effect of age at first mating on fertility \\ of young does and their first litter performance
}

Two hundred and ten does of the White of Dendermonde breed were used in this study.

They were presented for the first time to a male rabbit and distributed into three groups of age: $44 \mathrm{I} / 2$ or 5 mionths. During the rearing period a daily ration of $\mathrm{I} 5 \mathrm{O} g$ of a commercial rabbit feed was given to the first 25 does of each groups. The other 45 received $200 \mathrm{~g}$ according to our previous findings (VAN DEN BROECK and LAMPO, 1977). 


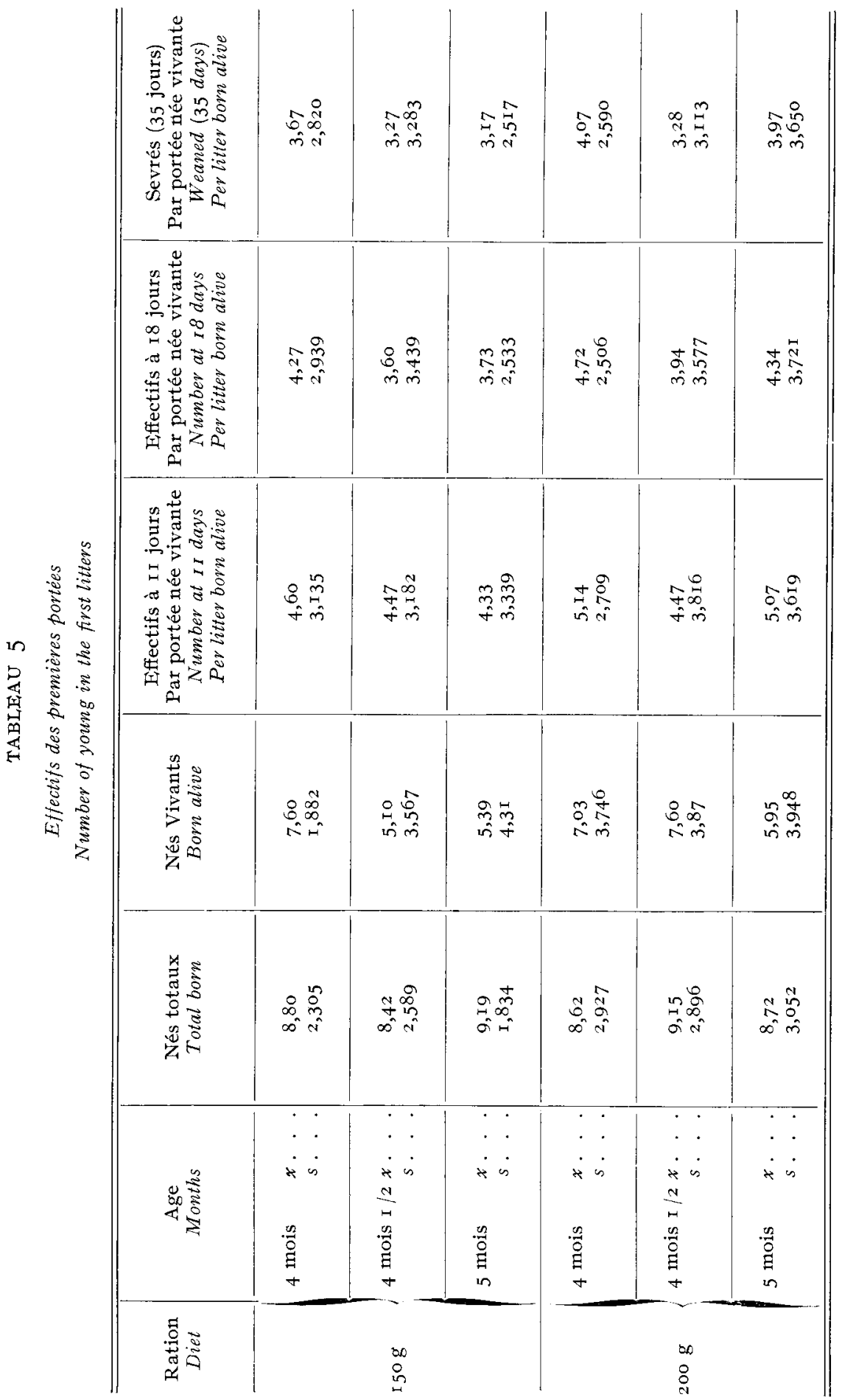




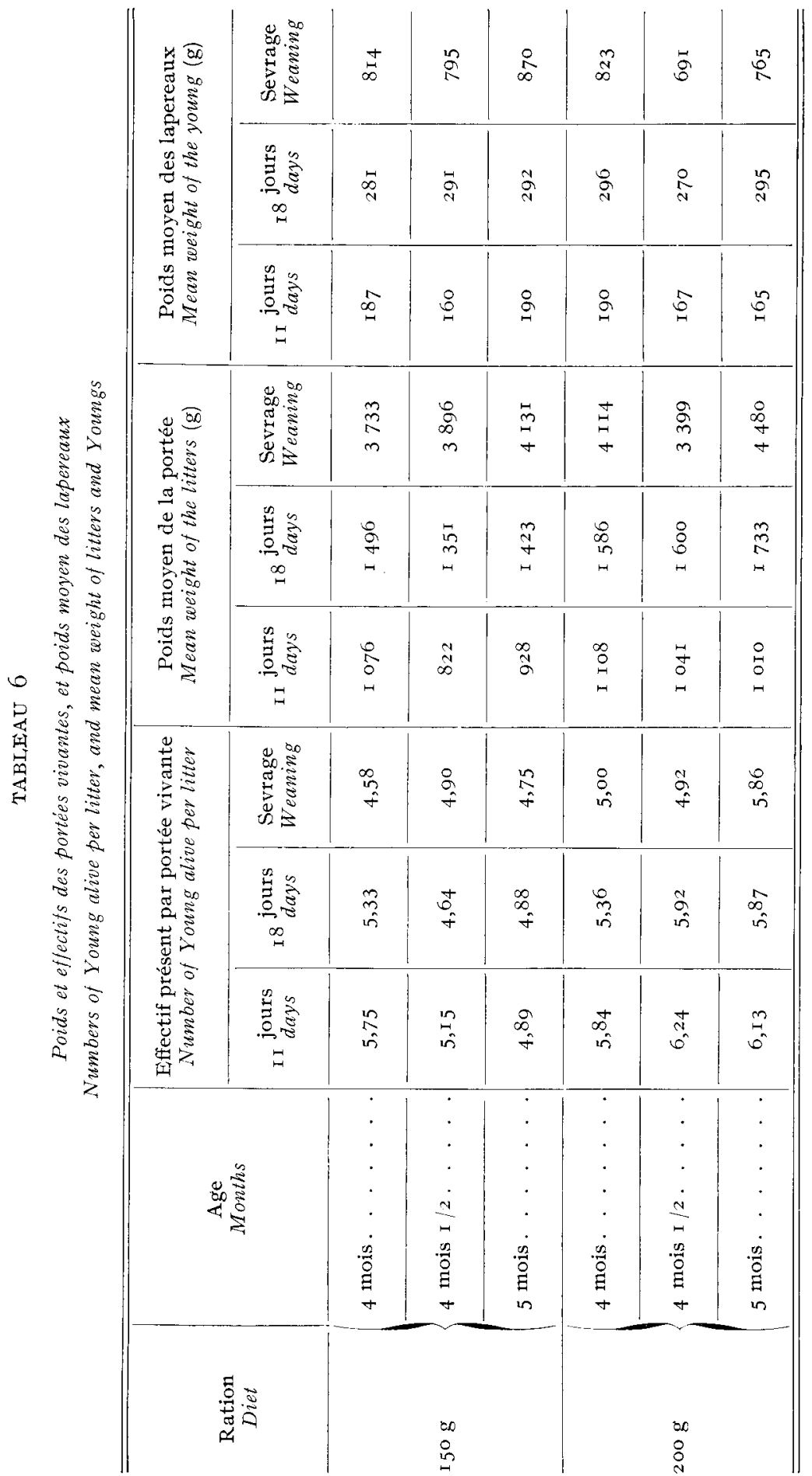



place.

A flushing of 4 days preceded the first mating-trial and was continued till copulation took

The distribution of the does over the seasons by order of selection day is given in table $\mathrm{I}$. Their weight on the day of mating is reported in table 2. There is a significant difference in body-weight between rations at each age, and also between the 4-5 age in each ration. Feed intake during tearing and flushing is given in table 3. During the flushing, does of the $150 \mathrm{~g}$ ration tried to compensate for their delay in growth by eating more than the double of their ration. This significantly exceeded the level of intake of the other ration. Table 4 shows the efficiency of first mating, including pregnancy-rate, nest-building and nursing-behavior. Mating occurred more frequently at the first confrontation with the male in the best fed group, especially in the eldest groups. These does were also more receptive, although the difference was not significant in the sub-groups.

Nest-building and nursing were definitely worse in the does of 5 months. In table 5 the number of young in the first litter are recorded at birth, I I, I 8 and 35 days. No differences are found. Considering only litters alive no difference in numbers nor in mean weights was observed (table 6).

In conclusion we may say at the age of 4 months the does were sufficiently fertile, had a good libido and an acceptable conception rate after first mating. Also the maternal abilities were developped, but tended to decrease with age. A very high mortality before weaning was observed in all groups, the explanation of which is not clear.

\section{Références bibliographiques}

Critchlov V., Bar-Sela M. E., 1967, dans Martini L., Ganong W. F. Neuroendocrinology, vol. II, Academic Press, New York and London, I967.

KALINOWSKI T., RUDOLPH W. I977. Reproduktionsleitstungen von Kaninchen bei Intensivhaltung. I. - Die Wurfgrösse des I. bis 6. Wurfes von Weissen Neusseländern. Wiss. Zeitschr. Wilh. Pieck Univ. Rostock, 26, Mat.-Nat., Heft I, 77-80.

LETEVRE Brigitte, MarTine'T L., Moret B., 1976. Environnement et comportement d'œestrus chez la lapine. Comm. no 6r, ier Congr. Int. Cunic. Dijon, France, $3 \mathrm{I}$ mars-2 avril $197^{6}$.

I,EFEVRE Brigitte, MORET B., I978. Influence d'une modification brutale de l'environnement sur l'apparition de l'œstrus chez la lapine nullipare. Ann. Biol. anim. Bioch. Biophys., 18, 695-698.

LEBAS F., I974. Effet de l'âge à la première saillie sur les performances de reproduction des lapines. Session d'information sur l'Alimentation et les Techniques d'élevage du lapin. I.T.A.V.I., Rambouillet, France 5 et 6 nov. 1974 .

Levasseur Marie-Claire, 1977. Toughts on Puberty. Initiation of the gonadotrophic function. Ann. Biol. anim. Bioch. Biophys., 17, 345-36r.

McDONALD L. E., I975. Veterinary Findocrinology and Reproduction. Lea and Febiger, Philadelphia, $492 \mathrm{pp}$.

MAR'TINFT I., I973. Physiologie de la Reproduction du lapin. Session d'information sur la reproduction et la sétection du lapin de chair. I.T.A.V.I., Toulouse, France, Io et II avril 1973.

PRUD'HON M., 1975. Fisiologia della riproduzione. Conoscerla a fondo per la sfruttarla meglio. Coniglicoltura, XII, ro, 35-4I.

Rouvier R., POUJARDieU B., VRILLon J. L., I973. Analyse statistique des performances d'élevage des lapines. Facteurs du milieu, corrélations, répétabilités. Ann. Génét. Sél. anim., 5, 83-I07.

TORRES S., 1977. Aspects physiologiques de la reproduction chez la lapine. Cuniculture, 4, I37-I 4 I.

VAN DEN BROECK Lea, LAMPO Ph., I977. Influence de trois niveaux d'alimentation de lapines futures reproductrices sur l'ardeur sexuelle et la fertilité à 4 mois. Ann. Zootech., 26, 565-574. 